\title{
Discussion of Shue et al. "Fat Injection: A Systemic Review of Injection Volumes by Facial Subunit"
}

\author{
Timothy Marten ${ }^{1}$
}

Received: 2 April 2018/ Accepted: 2 April 2018/Published online: 9 July 2018

(C) The Author(s) 2018

Level of Evidence $V$ This journal requires that authors assign a level of evidence to each article. For a full description of these Evidence-Based Medicine ratings, please refer to the Table of Contents or the online Instructions to Authors www.springer.com/00266.

\section{The Ascendency of Fat Grafting and the Coleman Technique}

Shue et al. rightly state in their interesting and ambitious article "Coleman popularized" fat grating, but this characterization falls short in describing the monumental contribution Coleman has made and the paradigm shift his teachings and advocacy have brought about in our approach to rejuvenation of the aging face, and rejuvenation and reconstruction of the breast and body. His work has come to influence almost every aesthetic and reconstructive procedure performed in the modern practice of plastic surgery. Coleman has shown us that "lifting" cannot correct all aspects of the aging change of the face and fat grafting allows treatment of age-associated loss of facial volume not addressed by traditional facelift surgery. Fat grafting comprises the "missing link" in facial

All descriptions of concepts, methods, and technique included in this article are courtesy of the Marten Clinic of Plastic Surgery and are used with permission.

Timothy Marten

tmarten@martenclinic.com

1 Marten Clinic of Plastic Surgery, 450 Sutter St, Suite 2222, San Francisco, CA 94108, USA rejuvenation, and Coleman's work stands as the most important new addition to facelift surgery since the introduction of the SMAS technique. The importance of his contribution cannot be over-stated.

\section{Fat Grafting as an Integral Part of Facial Rejuvenation}

Although Shue et al. state in the opening of their article that "the importance of volume changes in an aging face is well recognized" and that "fat grafting ... has become an integral component of aesthetic surgery", it should be noted that general acceptance of these notions is a relatively recent development and for much of its period of evolution fat grafting was viewed by many surgeons with suspicion, disbelief, and even hostility. Many physicians who engaged in rejuvenation of the face claimed fat grafting was "not necessary," "didn't work," "made the face look fat," was "lumpy," and so on. A surgeon's view of the utility of fat grafting and its success as a technique will also be unavoidably colored by our own biases-surgeons with filler bias will report poorer outcomes and more failures with fat grafting, whereas advocates of the technique may over-report success rates. It is also the case that one can't address problems one can't see.

\section{Assessing Outcomes of Facial Fat Grafting}

Although the human eye is notoriously flawed at recognizing and objectively quantifying volume, it is quite astute in subliminally recognizing and subjectively characterizing 
volume loss in the face. Patients with full faces and youthful distribution of facial tissues are easily recognizable as appearing young, fit, and healthy, whereas patients who are volume-depleted are instantly acknowledged and regarded as "old," unhealthy, or even ill appearing.

And although eventually beauty will prove amenable to scientific analysis and ultimately quantifiable, in this sense "success of intervention measured as patient satisfaction rather than quantitative analysis of volume change" is perhaps a legitimate way to define favorable outcomes. One must accept that fat grafting is a tool-not a guaranteed path to success-and that simply achieving a "volume change" on the face is not synonymous with producing or restoring a youthful, beautiful appearance. The surgeon performing fat grafting must recognize that without artistic vision there can be technical successes that are aesthetic failures.

It is also the case as Shue et al. point out in their review that "photographs can be misleading due to variability in film color, position, the presence or absence of flash, flash intensity, and facial expression." A photograph taken without flash accentuates facial hollowness and ages that subject, whereas a flash fills in facial hollows and provides a more flattering appearance. It is still often the case that we will see a before and after photograph pair presented at our scientific meetings or published in our journals (or posted on practice Web sites) in which the before photographs are taken without a flash, and the after with. In these cases, the "improvement" is illusorily, deceptive, even dishonest.

Finally, patients and surgeons are not always good at appreciating nuanced outcomes and it can take time for patients to appreciate their results and for the surgeon to refine her or his "artistic eye." It could be argued that some of the results shown in the accompanying special topic article that follows this discussion article are "overdone," but we have found we often have to show aggressively treated faces for the surgeon trying to learn the technique to see the results. It is also the case that the nuanced result will appear to the untrained eye as if "the fat went away." Once the concept is understood and the result can be recognized, one can then work toward refinement and nuance. Ultimately, success in fat grafting, at its best, is realized in the making of multiple nuanced changes over multiple areas of the face, and not spot filling of one or two areas.

\section{Defining Anatomic Regions}

Shue et al. state that "the anatomic specificity reported by each author varies greatly and limits comparison between studies" and this is indeed a valid consideration. Anatomic areas designated for treatment have not been precisely defined in the past and arguably do need some standardization if better and more scientifically meaningful comparisons are to be made in the future. In reality, these designations are somewhat arbitrary and hard to define, however-where does the cheek end and the mid-face begin? Where does the infra-orbital area end and the "tear trough" begin? And as the surgeon experienced in fat grafting is well aware all areas essentially overlap others and end up being feathered together when the procedure is properly performed. In Figs. 29-31 of the special topic article that follows this discussion, we endeavor to define the terms and anatomic definitions we have used and have found useful in our fat grafting procedures. Hopefully, these might serve as a starting point for the future creation and agreement on a uniform nomenclature.

\section{Deciding How Much Fat is Needed at Each Site}

The essential question Shue et al. try to answer in their review of available literature they have undertaken on facial fat grafting is how much fat needs to be injected in each area to obtain a predictable and specific result. And as their thoughtful analysis has shown, this is not a simple question to answer, and one in which multiple factors come into play. These may include what part of the body the fat is harvested from, the condition of the fat at the harvest site (previous SAL, cryolipolysis, other invasive and noninvasive fat reduction procedures), how the fat was harvested (type of instrumentation use, vacuum force applied, etc.,), how the fat was processed (centrifuging or filtering or straining, etc.), how the fat was injected (cannula size, particulate scatter or bolus injection), the condition of the tissues at the recipient site (previous SAL, cryolipolysis, chemolipolysis/deoxycholic acid, other invasive, and noninvasive fat reduction procedures), and perhaps even how the patient was managed postoperatively (avoidance of overly cold compresses and catabolic state, etc.,). It is clear the factors that might influence outcomes are many and varied.

It is also the case that the surgeon cannot simply rely on what she or he sees in the OR-that is, step up to the patient and begin injecting fat until the patient looks better (or even "over-corrected"), and making the decision as to how much fat to infiltrate intra-operatively. A better approach and a prudent and proven strategy for determining the amount of fat needed for a given site is to rate the severity of the atrophy for each region of the face based on what is seen in the preoperative photographs, and then to use published empirical data now available to choose the amount to be infiltrated into each area. This amounts to simply rating the severity of atrophy at each proposed site of treatment as "small," "medium" and "large," and then 
using data published regarding range of amounts typically needed as a guide for treatment of each area. If the defect is "small," one would choose an amount from the low end of the recommended range. If the defect is "large," one would choose an amount from the high end, and if "medium" somewhere in between. The authors' recommended ranges for the technique described appear in tables and Figs. 29, 30,31 of the accompanying special topic article.

The insightful surgeon can see that these parameters must be considered as guidelines and not absolutes and that considerations such as how the fat was harvested, how it was processed, how it was injected, what equipment was used, and the condition of the tissues receiving the fat as discussed above will all influence amounts needed and eventual outcomes.

Patients who are smokers or previous smokers and patients who have undergone previous noninvasive radiofrequency and ultrasonic "skin shrinking" procedures are likely to have compromised subdermal microcirculation and micro-lymphatic vessels, and as such will be less than optimal candidates for fat grafting and need more fat and several staged treatments than those who have not. Similarly, patients who are longstanding filler users, especially if they have used more inflammatory fillers such as PLLA, are likely to have internal facial fibrosis and inflammatory changes rendering them suboptimal candidates as well for fat grafting, and they exist as patients who will require that larger volumes be administered to obtain an equivalent result. Patients undergoing concomitant facelifts will typically require less fat than patients undergoing isolated fat grafting as their skin envelope is being reduced. It is also the case that the thoughtful injector must add a compensation for a large and small face, and adjust the amounts administered accordingly.

Ultimately, however, the strategy outlined above, and in particular the range of recommended volumes to administer, serves as a time-tested starting point and one that will shorten the beginning injector's learning curve, and serves as a point of reference from which one develops her or his own ranges with experience and over time. It should be understood that the volume ranges recommended are not absolutes and are not a guarantee of success. Nonetheless, this strategy has proven very useful in our own practices over several decades of combined experience and is the strategy we currently employ today. And as such, the surgeon currently seeking to incorporate fat grafting into her or his facelift procedures has a significant head start over their predecessors who had to develop these guidelines largely through trial and error.

\section{The Face Must be Recognized as a Sculptural Entity and Not Simply a Collection of Fat Compartments to be Filled}

Although we need to better understand the anatomy of the face and find ways to technically improve the fat grafting procedure, ultimately success will and can come only from viewing the face as a sculptural entity and whether its contours following the procedure are attractive and appealing to the eye, and not that a given area is simply larger. Fat should be placed where it is aesthetically indicated and where the artistically observant and trained eye says it should go. Fat should not arbitrarily be injected into a particular anatomic compartment, and fat compartment anatomy and fill should be considered subservient to overall facial contour. In many instances, fat will and should even be placed in areas it would not normally be found.

\section{Fat Grafting is More Than Rejuvenation: It Provides the Opportunity to Improve Appearance and Make Patients More Attractive}

Shue et al. state in their review that the "the goal of autologous fat grafting for an aging face is to create a natural, rejuvenated appearance," but fat grafting provides an opportunity to improve a patient's appearance above and beyond rejuvenation-it provides an opportunity to enhance a patient's appearance and make them more proportionate and beautiful. Fat grafting amounts to the opportunity to provide augmentation of the facial skeleton in the cheeks, submalar, infra-orbital, piriform, chin, and jawline areas and to perform the equivalent of cheek, submalar, "tear trough," peri piriform, chin, and jawline implants when indicated at a fraction of the time trouble and expense of placing such implants. Improvements in a patient's nasal appearance can add the equivalent of a nuanced rhinoplasty to the procedure. These are powerful advantages to the surgeon seeking to beautify, and not simply fill, their patient's faces.

\section{Fat Grafting While Straightforward is Not "Simple"}

Shue et al. refer to the fat grafting technique as "simple" in their review, but this is a misguided notion and one of the major impediments to success that the surgeon trying to learn the procedure will encounter. For the procedure to be successful, fat must be harvested in a specific time-consuming manner, and it must then be processed and 
infiltrated in a technically and artistically demanding and time-consuming process. It must also be recognized that atrophy is generally a pan-facial process and that fat grafting is most effective and most properly applied when multiple areas are treated. If one acknowledges that 20-40 back-and-forth cannula movements are needed to empty a 1-cc syringe, it can be seen that it can take 1 min or more to administer $1 \mathrm{cc}$ of fat and that that in turn can translate into $30-90 \mathrm{~min}$ or more of time spent injecting the 30-90 cc of fat that is often needed in the facelift patientnot including time spent harvesting and processing the fat. Time must therefore be planned appropriately, and the surgeon must work in a well-organized and efficient fashion. Underestimating technical and artistic difficulty of fat grafting is one of the most common causes of treatment failures.

\section{Isolated Facial Fat Grafting and Fat Grafting Performed Simultaneously with a Facelift are Not the Same Thing and Can't be Directly Compared}

There are distinct advantages to performing fat grafting in conjunction with a facelift rather than as an isolated procedure, and the difference must be considered in literature reviews such as the one undertaken by Shue et al. if meaningful conclusions are to be drawn regarding the questions they are trying to answer. When a facelift is performed in conjunction with fat grafting, one is repositioning ptotic facial tissues and reducing the size of the facial skin envelope-maneuvers that in and of themselves improve facial contour and markedly reduce the amount of fat needed to achieve the intended result. When isolated fat grafting is performed, one must overcome tissue ptosis and compensate for a larger skin envelope by administering larger volumes of fat. The result is often a smoother and less hollow-appearing face, but one that is abnormally large and unfeminine appearing, and one that lacks youthful contour and lightness in appearance.

\section{Fat Grafting Should be Viewed as an "Art Project" and Not a "Science Experiment"}

Artistic vision is not intuitive for most plastic surgeons and something that is not specifically developed or taught in our training programs. We exist and function in a world of science and technique and in time become comfortable in that world, in holding that worldview, thinking in that way, and using the related vernacular. We speak in scientific terms and strive to develop a scientific basis for the things we do, and this is indeed a powerful approach to caring for many problems faced by plastic surgery patients. Too often however, surgeons become primarily focused on science when it comes to fat grafting, as "science"-related terms and concerns are things we know and are comfortable with. As a result, the well-meaning surgeon who is uncomfortable with the "art" aspect of the procedure can end up hiding from the fact that fat grafting is an artistic endeavor by becoming hyper-focused on technique.

Fat grafting should be viewed more as an art project than a science experiment, and the surgeon who endeavors to succeed in fat grafting must approach the process more as an artist and less as a scientist. A painter for the most part will be largely unconcerned with the chemical composition of her or his paint-she or he just wants paint to put on the canvas. And if paint is given to a group of people who aspire to be painters, only a handful of them will have the instincts and wherewithal to use it in an artistic manner. Obsessing about brush bristle type or the chemical composition of paint rarely makes one into a good painter.

Success in fat grafting must ultimately be measured artistically and not as simple technical success in adding fat to the face. The real challenge is to create beauty, not to simply add fat to the face, and "unsatisfactory results" are largely artistic failures that cannot be overcome by technical focus alone.

\section{Fat Retention}

Shue et al. state that "fat injection(s) (are) unpredictable (and) resorption is still a major limitation" and this has been the conventional wisdom and the naysayer's mantra throughout the development and evolution of the fat grafting technique. Patients seeking the procedure and surgeons striving to learn the fat grafting technique are often pre-occupied with the question of "how much of the fat survives?" And at first thought, this seems germane-if one knows how much of the fat survives, one could then over-correct by a similar amount and ostensibly obtain the desired result. But what really matters to the clinician treating the patient is what amount of fat must be administered to achieve the result the patient and the surgeon want. In such a situation, empirical information is more useful and empowering than a simple knowledge of what the survival rate is, and it is what really matters. A better question to try to answer is "how much fat is needed to get the desired result?" not "how much fat survives?"

Resorption is a factor in all grafts plastic surgeons perform and not a legitimate reason to rebuke the fat grafting procedure. Some tissues are durable and graft reliably with high "take." These include skin grafts and hair transplants. Other tissues are more fragile, and the "take" is less. These include bone grafts, nerve grafts, and fat grafts. But simply needing to put in more bone as a graft than will be present 
in the healed state does not negate the utility of the bone graft procedure. The same is true with fat-the fact that some fat is undeniably lost does not negate the ultimate success and utility of the procedure.

"Unpredictability" is also a common refuge for the fat grafting doubter, and while Shue et al. have reviewed a large series of published articles, their review does not address the relative predictability of fat grafting relative to other aesthetic surgery procedures we perform. And at least in our practices, fat grafting has come to represent one of the more predictable procedures we perform and we feel the technique has evolved and matured and now falls into alignment with almost all other aesthetic surgery procedures plastic surgeons perform as far as touch-ups and revisions are concerned. In fact, our experience seems to indicate that we perform fewer revisions and touch-ups on fat grafting procedures than the generally accepted revision rates for rhinoplasties, breast implants, liposuction, abdominoplasty, body lifts, and the like. Revisions and touch-up on these procedures can exceed 20-30\%. This has not been the case with our fat grafting procedures. Once experience is gained with fat grafting-we now have the needed equipment and know how to perform the procedure in a predicable way - the revision and touch-up rate will be seen to be less for fat grafting than for these other procedures.

The most common reason for a patient wanting a second-stage fat grafting procedure is not a problem or complication, but their desire for a bit more of a good thing. This is not a failure of treatment, and it is merely good practice to treat patients conservatively. It is easier to add a bit more in a second stage than to be removing fat that was too aggressively placed in the primary procedure. And while fat grafting for many patients will be a straightforward one-stage procedure, it is prudent to present it to patients as a possibly staged procedure.

\section{Many Patients have Compromised Faces and Must be Expected to be More Difficult to Treat Satisfactorily in a Single Stage}

Not all patients are equivalent, and many presenting for fat grafting will not have "virgin" faces and as such are suboptimal candidates for the procedure and are patients who may require several stages of treatment to achieve the result they desire. These include patients who presently use fillers or have used them in the past, patients who have previously undergone facial liposuction, and patients who have undergone ultrasonic or radiofrequency "skin shrinking" treatments, facial cryolipolysis, chemolipolysis, or like "noninvasive" treatments. In the current social climate we live in today, this amounts to many patients.
As Shue and others have stated patients using fillers are difficult to assess "moving targets," and it can be difficult to know what is their face and what is filler. Often there is much more filler present than patients recall receiving, and often it was injected by multiple physicians making it difficult to obtain records of what has been done. Even when records can be obtained, they are often not detailed and can be difficult to interpret. It is also the case that many patients will not be forthcoming about the extent of their filler use fearing that they might be rejected as fat grafting patients. It is likely often the case that when a surgeon treats these patients and they return for a "touch-up" of their fat grafting the surgeon is actually just replacing filler that was preoperatively present but was lost over time. Once this dynamic is understood, it becomes clear why it is prudent to have a policy that charges will apply for all touch-up and secondary procedures that are needed or wanted.

Filler also likely compromises outcomes by acting as a physical barrier to neovascularization of fat grafts as to optimally take and survive the injected fat needs contact with perfused tissue. Even if HA fillers are dissolved enzymatically, inflammation is likely present for an extended period of time thereafter and likely results in compromised outcomes if subsequent fat grafting procedures are preformed. Non-HA fillers and highly inflammatory fillers such as PLLA represent a more difficult and perplexing problem in that they result in chronic inflammation and internal fibrosis and make the take of fat grafts even less reliable. Patients who have undergone filler treatments are more likely to have suboptimal graft survival, uneven take, and need multiple staged treatments, and patient filler use is a variable that must be considered in future reviews of the literature on this subject.

The patient who has undergone facial ultrasonic and radiofrequency "skin shrinking" treatments is part of a growing body of patients who seen to sustain some compromise of their microcirculation and superficial microlymphatic system as a result of these and like treatments that puts them at greater risk of slough and healing-related problems following facelift, and suboptimal take when fat grafting is performed. In these procedures, energy is directed and dissipated under the skin, rather than on its surface, and energy meant to "tighten" the face appears to damage both subdermal microcirculation and lymphatics, and adjacent subcutaneous fat. The resulting atrophy is typically pan facial and irregular and can be very difficult to treat. The incidence of problems seems to parallel the number and intensity of treatments the patient has undergone which one would expect to parallel the degree of compromise to their microcirculation. These patients also seem to have some lymphatic compromise as well, which leads to prolonged edema and compromised take of fat 
grafts. Patients who have previously undergone "skin tightening" procedures should be informed that they are more likely to have suboptimal graft survival, uneven take, a longer period of recovery, and need multiple staged treatments, and these sorts of treatments must be considered as a variable effecting outcomes in future reviews of the literature on this subject.

\section{Learning the Procedure}

Success in fat grafting will ultimately only come from training one's artistic eye and not from simply engaging in a series of recommended technical steps. This is no small undertaking for surgeons trained and traditionally focused on science and technique, and in our current educational climate that provides little training in the way of artistic and aesthetic goals. It is not possible to "standardize" the procedure, and each surgeon must ultimately embark upon her or his own journey to train their artistic eye and gain mastery of the technique. Published parameters must be regarded as guidelines and not absolutes, and as Shue et al. state "must be adjusted based on each patient's unique needs."

So how does one go about learning this procedure and how much fat to put where? First, one must acknowledge atrophy as a part of the aging change of the face and learn to recognize it. One must set aside unfounded fears perpetuated by those who don't understand the need for the procedure or who are unwilling to take the time to learn it and do it. One must learn the basics of the fat grafting technique and obtain the needed equipment to properly harvest, process, and inject fat. One must accept that fat grafting is a tool-not a guaranteed path to success and that it will only be beneficial if carried out carefully and correctly. One must make the needed commitment of time in the OR to properly perform the procedure and not underestimate its technical and artistic difficulty. One must recognize that without artistic vision there can be technical successes that are aesthetic failures. One should recognize that it is best to make small additions of fat to the face at first to gain familiarity with the technique. Starting out conservatively will mean that problems, should they occur, will be minor and easily managed. Patients should then be followed closely and outcomes critically analyzed by taking well-matched follow-up photographs. Such analysis will allow one to figure out what came out well and what did not, and to then strive to do the next case better.

As Shue et al. assert, that despite its inherent shortcomings "nonetheless, the findings from this paper can serve as a starting point for the less experienced surgeon, a base for research, and a clinical estimate" as to where, how, and how much.

\section{Compliance with Ethical Standards}

Conflict of interest The author declares that he has no conflict of interest to disclose. 